\title{
Intervención sobre el funcionamiento ejecutivo en un caso de TDAH: implicaciones en conciencia fonológica y lectura
}

\section{Intervention on executive functioning in a case of ADHD: implications for phonological awareness and reading}

\author{
Nuria Pérez Mariño \\ Universidade da Coruña
}

\begin{abstract}
Resumen
El Trastorno por Déficit de Atención con Hiperactividad (TDAH) es uno de los trastornos del neurodesarrollo más frecuentes de la infancia, caracterizándose, principalmente, por un fallo en las funciones ejecutivas. Frecuentemente aparecen asociadas dificultades en la lectura que podrían atribuirse al fallo en las funciones ejecutivas, siendo posible que exista una relación entre éstas y un bajo desempeño en tareas de conciencia fonológica. El objetivo principal del trabajo fue valorar los efectos de un programa de intervención centrado en el funcionamiento ejecutivo sobre las dificultades en conciencia fonológica y en lectura en un niño de 7 años con TDAH.

Palabras clave: TDAH, funciones ejecutivas, memoria de trabajo, velocidad de procesamiento, conciencia fonológica, habilidades lectoras.
\end{abstract}

\begin{abstract}
The attention deficit hyperactivity disorder (ADHD) is one of the most common neurodevelopmental disorders of childhood characterized mainly by a failure in executive functions. Often associated reading difficulties which could be attributed to failure in executive functions, being possible a relationship between them and poor performance in phonological awareness. The main objective of this study was to assess the effects of an intervention program focused on executive functioning over phonological awareness and reading in a 7 year old boy with ADHD.

Keywords: ADHD, executive functions, working memory, processing speed, phonological awareness, reading skills.
\end{abstract}

\section{Introducción}

Desde mediados del siglo XIX existen descripciones y trabajos sobre el TDAH, sufriendo su denominación múltiples cambios a lo largo de la historia debido a las variaciones en la forma de entender dicho trastorno. En la actualidad, se define el TDAH como un trastorno neurobiológico de carácter crónico, sintomáticamente evolutivo y de probable transmisión genética. Se caracteriza por una deficiente capacidad de inhibición y dificultades tanto para mantener la atención como para autorregularse presentándose tanto frente actividades académicas como cotidianas (García, 2014).

Resulta improbable encontrar una única causa que explique el TDAH, siendo más bien resultado de una serie de vulnerabilidades biológicas que interactúan entre sí y con otras variables ambientales. En los últimos años se ha ido concediendo más importancia a las funciones ejecutivas, considerándose el TDAH un trastorno que deriva de un fallo en las mismas. A pesar de no existir consenso sobre todos los procesos que conforman las funciones ejecutivas, se suelen incluir la atención, inhibición, regulación y selección de conductas, planificación, organización y flexibilidad (Abad-Mas et al., 2011; García et al., 2014).

$\mathrm{Si}$ bien las funciones ejecutivas pueden estudiarse desde una aproximación puramente funcional, considerar un sustrato anatómico proporciona información valiosa respecto a su organización y funcionamiento. Algunas investigaciones apuntan hacia la importancia del área prefrontal derecha en el control de las respuestas inhibitorias, del área prefrontal dorsolateral izquierda en la modulación de la atención selectiva, así como de un factor motivacional que juega también un papel importante dentro de las funciones ejecutivas, asociando éste a la corteza orbitofrontal (Abad-Mas et al., 2011; García et al., 2014).

El rendimiento en la lectura está ligado al buen funcionamiento ejecutivo, siendo la memoria de trabajo una de las funciones principales para lograr un buen desempeño lector (Miranda-Casas, Fernández, Robledo y García-Castellar, 2010).

La lectura conlleva, numerosas operaciones mentales dirigidas a interpretar y comprender un texto. Es importante tener en cuenta que un niño puede decodificar correctamente las palabras y aún así una vez leído un texto no haber entendido nada. Esto puede llevar a equívocos al considerar que el problema reside en la decodificación de las palabras, cuando puede deberse a las dificultades en memoria de trabajo. Las dificultades para autorregularse y la impulsividad pueden causar que durante la lectura no se asegure de si una palabra leída encaja con el contenido del texto que está leyendo, sustituyendo la misma por una de uso más frecuente. Esto puede provocar también dificultades o errores en la comprensión. Además, dificultades en planificación, inhibición de estímulos irrelevantes y memoria de trabajo hacen que resulte complicado mantener la información, sintetizarla y llevar a cabo los mandatos de manera ordenada (Martínez, 2014; Miranda-Casas et al., 2010).

La adquisición de la lectura parece relacionarse además con la conciencia fonológica. Ésta última presenta un carácter progresivo siendo un continuo en el que están implicados diferentes niveles y etapas evolutivos (Aguilar, Marchena, Navarro, Menacho y Alcalde, 2011).

La relación entre lectura y conciencia fonológica es bidireccional. La evidencia científica ha demostrado que 
déficits en las funciones ejecutivas se relacionan con dificultades en la adquisición de la lectoescritura. Es posible que puedan relacionarse también con un bajo desempeño en tareas de conciencia fonológica, debido a que estas requieren de un costo cognitivo, como mantener un estímulo en la memoria de trabajo, seleccionar información, inhibir lo irrelevante y mantener la atención en la tarea principal (Martínez, 2014).

Algunos trabajos afirman que las funciones ejecutivas tienen un peso mayor en el desarrollo de tareas de comprensión y velocidad lectora más que en tareas de decodificación, siendo considerado el proceso perceptivo como un factor menor ( Miranda-Casas et al., 2010).

En el presente trabajo se presenta un estudio de caso llevado a cabo con un niño de 7 años con TDAH. El objetivo ha sido comprobar la posible relación entre funcionamiento ejecutivo, conciencia fonológica y aprendizaje lector. El estudio se desarrolló a lo largo de tres fases: una evaluación previa a la intervención en la que se recogieron datos acerca de las variables de interés, una segunda fase que consistió en un entrenamiento en funciones ejecutivas $\mathrm{y}$, finalmente, una tercera fase consistente en una evaluación realizada al finalizar la intervención para comprobar si se habían producido cambios en las variables objeto de estudio. A continuación se describe cada una de estas fases, así como los resultados obtenidos.

\section{Método}

\section{Participante}

En el presente trabajo participó un niño, G., de 7 años y 7 meses de edad que actualmente cursa $1^{\circ}$ de Educación Primaria tras repetir tercero de infantil. Fue diagnosticado de TDAH con la presentación clínica combinada refiriendo además retraso madurativo.

En la actualidad el niño presenta comportamientos disruptivos, como agresividad y rigidez que dificultan su relación con otros niños y con los propios padres que, según comentan, son incapaces de controlarlo generándoles un sentimiento de frustración.

Presenta dificultades en el rendimiento escolar, fundamentalmente en la lectura, por lo que acude a intervención logopédica. Destacar que hasta el momento no se había realizado ninguna evaluación y la intervención se basaba fundamentalmente en la realización de tareas de lectoescritura. Mencionar además que durante las fases de evaluación e intervención el niño no recibía medicación.

\section{Instrumentos}

En el presente trabajo se emplearon diversos instrumentos tanto en las fases de evaluación como de intervención, los cuales serán descritos a continuación.

En la fase de evaluación previa a la intervención se utilizaron los siguientes instrumentos:

El Childhood Executive Functioning Inventory CHEXI- (Thorell y Nyberg, 2008) es un cuestionario que deben cumplimentar los padres o maestros. Permite valorar la memoria de trabajo, el control inhibitorio, la regulación y la planificación. Las puntuaciones próximas a la máxima son indicativas de dificultad.

La Batería de evaluación de los procesos lectores revisada -Prolec-R- (Cuetos, Rodríguez, Ruano y
Arribas, 2007) evalúa los procesos lectores para identificar dificultades en el aprendizaje de la lectura en niños de entre 6 y 12 años. Cuantifica el número de aciertos y errores y en algunas pruebas el tiempo invertido en completar la tarea.

El LolEva: del lenguaje Oral al Lenguaje Escrito (Fernández-Amado, Mayor, Zubiauz, Tuñas y Peralbo, 2006; Mayor et al., 2011) es una prueba informatizada que permite evaluar la conciencia fonológica y la competencia lectora inicial en niños de Educación Infantil y primer ciclo de Primaria.

Finalmente, se utilizaron las pruebas corsi para valorar la memoria de trabajo visoespacial en orden inverso y letterdigit para valora la velocidad de procesamiento del Psychology Experiment Building Language -PEBL(Mueller y Piper, 2014).

Dichos instrumentos fueron utilizados para valorar los resultados tras finalizar la intervención, a excepción del CHEXI, ya que no se esperaban cambios en los datos obtenidos con el mismo debido a la brevedad de la intervención (un mes y una semana).

En la fase de intervención se emplearon para diseñar las sesiones el Programa para la atención educativa al alumnado con TDAH (Artiles y Jiménez, 2006) y el Programa de intervención cognitivo-conductual para niños con déficit de atención con hiperactividad (Orjales, 2001). Ambos tienen como objetivo general mejorar las funciones ejecutivas ofreciendo diversas actividades. Debido a que en este caso se iba a trabajar de manera individual y el tiempo disponible en cada sesión era menor al planteado por los programas se realizaron algunas modificaciones y se introdujeron actividades diferentes para trabajar aquellas habilidades en las el niño que presentaba mayores dificultades.

\section{Procedimiento}

Como ya se mencionó anteriormente, el trabajo se llevó a cabo a lo largo de tres fases: evaluación previa a la intervención, intervención propiamente dicha y evaluación posterior a la intervención.

La fase de evaluación previa a la intervención se desarrolló a lo largo de tres sesiones, con una duración de 60 minutos cada una. En la primera sesión se aplicó el $P R O L E C-R$, seguido de la aplicación del LOLEVA en las dos siguientes. El último día de evaluación se administró, además del LOLEVA, las pruebas corsi y letterdigit del PEBL. Se entregó, además, a los padres del niño el cuestionario CHEXI para que lo cumplimentaran y poder obtener así más información sobre el mismo.

Tras realizar la evaluación anteriormente descrita se procedió con la fase de intervención, la cual se desarrollo a lo largo de ocho sesiones de una duración de 60 minutos cada una de ellas, trabajando con el niño de manera individual dos veces por semana (lunes y viernes generalmente). El horario de todas las sesiones fue siempre el mismo (18.00 horas).

Los objetivos que se pretendían alcanzar realizando una intervención centrada en el desarrollo de las funciones ejecutivas fueron:

- Mejorar la memoria de trabajo.

- Disminuir el tiempo de respuesta.

- Mejorar la conciencia fonológica. 
- Mejorar las habilidades lectoras.

Se establecieron una serie de normas dirigidas a mejorar el comportamiento del niño durante la sesión. En función del cumplimento de las mismas el niño obtendría puntos que le permitirían alcanzar diversos premios pactados en un inicio. Para que el niño consiguiera mayor autonomía comportamental se utilizó la "autoevaluación reforzada", para que evaluara su propia conducta y se otorgase los puntos en función del cumplimiento de las normas. Se utilizaron también las autoinstrucciones y la técnica "¡STOP! párate y piensa" para que recordase los pasos que debía seguir para completar las diferentes tareas, y evitar las respuestas impulsivas.

Antes de cada sesión se recordaban las normas, las autoistrucciones y la ficha de "¡STOP! Párate y piensa". Tras ello se procedía con la realización de las diversas actividades preparadas para cada sesión.

Al finalizar el programa descrito, se procedió con la fase de evaluación posterior a la intervención que se llevó a cabo en tres sesiones de 60 minutos cada una, distribuyéndose la aplicación de los diversos instrumentos al igual que en la fase de evaluación previa.

\section{Resultados}

Tras finalizar el programa de tratamiento y realizar la evaluación posterior a la intervención se procedió al análisis de los resultados, haciendo una comparación entre los datos obtenidos en la evaluación pre y post-intervención. A continuación se describen los resultados obtenidos con cada una de las pruebas.

En el cuestionario CHEXI realizado por los padres del niño las puntuaciones se situaron próximas a la máxima en todas las variables, siendo indicativo de dificultades. En control inhibitorio obtuvo una puntuación de 27 de 30 posibles y en regulación 20 de 25 puntos, siendo los procesos que presentan mayores dificultades. En planificación obtuvo 12 de 20; y en memoria de trabajo 35 de 45 posibles.

En la escala de índices principales del PROLEC-R las puntuaciones directas obtenidas en las subpruebas de nombre de letras $(\mathrm{PD}=26)$, igual-diferente $(\mathrm{PD}=9)$, lectura de pseudopalabras $(\mathrm{PD}=29)$, estructuras gramaticales $(\mathrm{PD}=10)$, comprensión de oraciones $(\mathrm{PD}=12)$ y comprensión oral $(\mathrm{PD}=0)$ fueron indicativas de dificultad; logrando puntuaciones consideradas dentro de la normalidad en las subpruebas de lectura de palabras $(\mathrm{PD}=29)$, signos de puntuación $(\mathrm{PD}=4)$ y comprensión de textos $(\mathrm{PD}=8)$. Una vez realizada la intervención los resultados mostraron una notable mejora, logrando puntuaciones consideradas dentro de la normalidad en todas las subpruebas.

En la escala de índices de precisión del PROLEC-R obtuvo, en la evaluación pre-intervención, dificultades severas en las subpruebas de lectura de palabras $(\mathrm{PD}=27)$ y lectura de pseudopalabras $(\mathrm{PD}=20)$. En el resto de subpruebas, nombre de letras $(\mathrm{PD}=17)$ y signos de puntuación $(\mathrm{PD}=6)$, logró puntuaciones situadas dentro de la normalidad, siendo la subprueba igual-diferente calificada como dudas. En la evaluación post-intervención en la subprueba de lectura de palabras $(\mathrm{PD}=33)$ progresó desde dificultades severas a dudas. En lectura de pseudopalabras $(\mathrm{PD}=31)$ alcanzó el nivel de normalidad. Las restantes pruebas, a excepción de igual-diferente $(\mathrm{PD}=16)$, calificada como dudas, se enmarcaron dentro de la normalidad.

En la escala índices de velocidad del PROLEC-R obtuvo, en la evaluación previa, una velocidad muy lenta en la subprueba nombre de letras $(\mathrm{PD}=65)$, siendo la velocidad en las restantes normal. En la evaluación posterior se redujo el tiempo empleado para realizar todas las pruebas estableciéndose además todos las puntuaciones dentro de la normalidad (ver Figura 1).

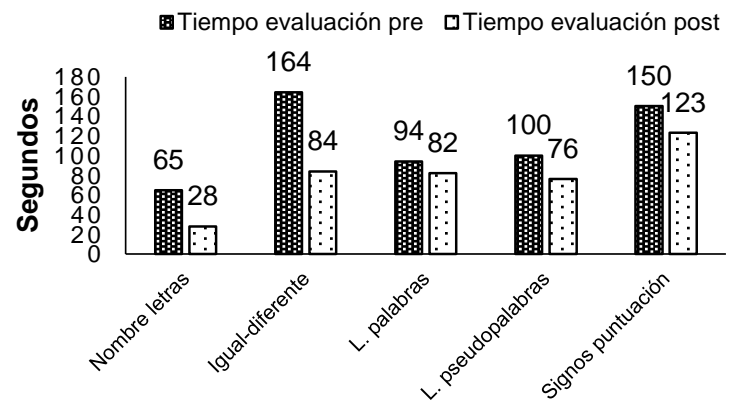

Figura 1. Resultados pre y post-intervención en la escala Índices de Velocidad del PROLEC-R.

Por lo tanto, los resultados obtenidos con el $P R O L E C-R$ muestran una mejora en todos los índices evaluados con este test una vez finalizado el período de intervención.

Los resultados obtenidos en las subpruebas de lectura del LOLEVA no muestran cambios en el número de aciertos y errores en la lectura de mayúsculas (27 aciertos de 29), minúsculas (25 de 29), palabras regulares, palabras complejas y pseudopalabras (realizando los 10 ítems de manera correcta), excepto en la subprueba de separación de frases obteniendo 2 aciertos en la evaluación previa y 4 en la evaluación posterior de los 5 posibles. Además, se observaron cambios en el tiempo empleado para realizar las diferentes tareas (ver Figura 2), reduciéndose en todas las subpruebas excepto en separación de frases, cuestión que puede deberse a que en la primera evaluación el niño realizó la tarea sin leer apenas las frases, dando respuestas precipitadas.

BTiempo evaluación pre aTiempo evaluación post

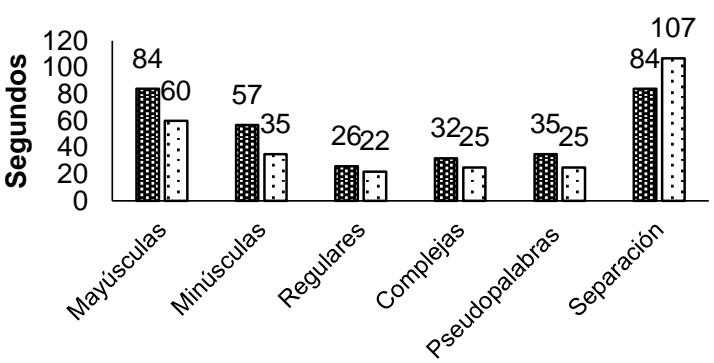

Figura 2. Comparación entre el tiempo empleado en la evaluación previa y posterior en las subpruebas de lectura del LOLEVA.

Se empleó, además, el LOLEVA para valorar la conciencia fonológica. Los resultados obtenidos en la evaluación realizada en la fase previa a la intervención y los obtenidos tras la realización de la fase de intervención muestran una mejora en la puntuación total de cada subprueba, excepto en omisión de sílaba final en la que 
no se produjeron cambios positivos ( 2 aciertos evaluación previa, 1 acierto evaluación posterior). En la Figura 3 se exponen gráficamente los resultados obtenidos en cada una de las subpruebas en ambas fases de la evaluación. Como puede comprobarse, en la subprueba de rima pasó de 4 aciertos en la evaluación previa a 8 en la posterior siendo la máxima 10. En las restantes subpruebas la puntuación máxima alcanzable es de 5, logrando estos en identificación de fonema final en la que pasó de 1 a 5 aciertos. En las subpruebas identificación de sílaba inicial, adición de sílaba inicial, identificación de fonema inicial y adición de fonema inicial pasa de 2 a 4 aciertos. En identificación de sílaba final y adición de sílaba final pasó de 3 a 4 aciertos; y en omisión de sílaba inicial, de 1 a 2 . En la subprueba adición de fonema final pasó de 1 a 4 aciertos, en omisión de fonema inicial de 0 a 2 y en omisión de fonema final de 0 a 4.

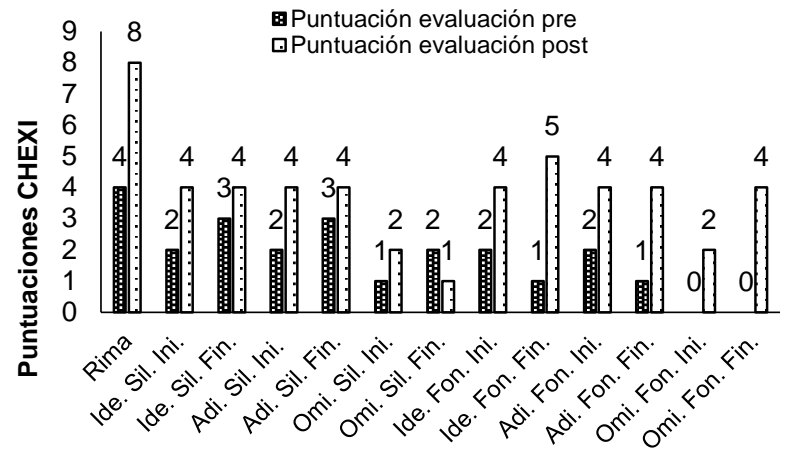

Figura 3. Comparación entre la puntuación de la evaluación pre y post-intervención en las subpruebas de conciencia fonológica del LOLEVA.

Los resultados obtenidos con el "corsi" de la batería $P E B L$ mostraron una progresión favorable en memoria de trabajo. En la evaluación previa el niño realizó 7 ensayos, recordando un máximo de 3 bloques en las secuencias y obteniendo una puntuación en memoria de trabajo de 3; mientras que en la posterior realizó 13 ensayos, recordando un máximo de 6 bloques y logrando una puntuación en memoria de trabajo de 5 .

El tiempo de respuesta medio empleado en la realización de la prueba "letterdigit" de la batería PEBL disminuyó ligeramente, pasando de 4193.67 a 3427.43 ms siendo el número de respuestas correctas elevadas tanto en la evaluación previa (29 de 30 ), como en la posterior (28 de 30$)$.

\section{Discusión y conclusiones}

La valoración y comparación de los resultados alcanzados antes y después del programa de intervención aplicado permitió observar cambios positivos en todas las variables analizadas.

Diversos estudios hablan de la importancia de la memoria de trabajo verbal, considerando ésta una capacidad que puede predecir el rendimiento en tareas de lectura. El déficit en memoria de trabajo se asociaría a mayores problemas de aprendizaje y menor rendimiento académico (Booth, Boyle y Kelly, 2014).

El aumento en el tiempo de respuesta parece relacionarse con la inatención y se asocia además con deficiencias en lectura de palabras, fluidez y comprensión (Jacobson et al., 2011).
Katz, Brown, Roth y Beers (2011) encontraron que las personas con TDAH y dificultades de lectura tenían más dificultades en velocidad de procesamiento y memoria de trabajo que niños con TDAH sin dificultades en lectura.

Las dificultades para identificar palabras iguales o diferentes y la realización de la lectura de palabras mejor que la de pseudopalabras podrían indicar que el niño lee mayormente a través de la ruta léxica. Dicha estrategia serviría tan sólo para leer palabras conocidas y puede fallar cuando se introduce un pequeño cambio en una grafía. Además, la atención es también un proceso clave en la realización de estas tareas. Esto coincide con lo encontrado en algunos estudios que afirman que existe una alta correlación entre las dificultades en lectura y la falta de atención. Las dificultades para autorregularse y la impulsividad pueden causar, además, que durante el proceso de lectura los niños no se detengan a corroborar si una palabra es o no correcta, sustituyéndola por otra más conocida, dato que podría relacionarse con las dificultades encontradas en la precisión lectora (Greven, Rijsdijk, Asherson y Plomin, 2012).

Las dificultades en comprensión de textos pueden deberse no solo a la complejidad de las estructuras gramaticales, sino también a la falta de atención sostenida durante la realización de la lectura y/o a los problemas para retener información debido a las dificultades en memoria de trabajo. Estas situaciones pueden llevar a equívocos, ya que no es la comprensión la que está afectada en sí misma (Miranda-Casas et al., 2010)

En conciencia fonológica el niño tuvo mayores dificultades en la evaluación previa en las subpruebas de fonemas. La conciencia fonológica presenta un carácter progresivo, siendo esta un continuo en el que están implicados diferentes niveles y etapas evolutivos. Sobre los 3 años los niños reconocen palabras que riman, adquiriendo la habilidad para identificar fonemas sobre los 8 (Aguilar et al., 2011). Por tanto, el niño es capaz de realizar de manera más satisfactoria tareas que se consideran más simples. Tras la intervención mejoró notablemente en la manipulación de fonemas. De hecho, no obtuvo cambios tan positivos en omisión de sílaba inicial y final. Esto último podría deberse a que eran éstas las últimas pruebas en aplicarse durante la evaluación por lo que el niño podría prestar menos atención y responder de manera más impulsiva conllevando así peores resultados.

El buen funcionamiento de la conciencia fonológica parece relacionarse con la capacidad para inhibir estímulos irrelevantes que permita al lector centrarse en lo importante, la memoria de trabajo que facilite mantener una serie de información en la mente para integrarla posteriormente $\mathrm{y}$ formar una secuencia temporal y las habilidades de autorregulación que permitan planificar y organizar la información de manera coherente (Miranda-Casas et al., 2010).

A pesar de no haber evaluado variables como la atención, planificación, etc. se pudo observar como el niño mejoraba en estas habilidades, resultándole más sencillo abordar las tareas propuestas a lo largo del período de intervención. 
Para finalizar y como conclusiones decir que: - Características del TDAH como las dificultades en inhibición de estímulos irrelevantes, los déficits en atención y la impulsividad dificultan la realización de tareas de lectura y conciencia fonológica. - Tras valorar los resultados alcanzados en la intervención se puede concluir que un programa de intervención diseñado para estimular las funciones ejecutivas en niños con TDAH y dificultades en conciencia fonológica y lectura ayuda a mejorar dichas habilidades. Aun así, los resultados deben tomarse con cautela, ya que se trata de un trabajo con un único caso, por lo que sería recomendable llevar a cabo más trabajos en esta línea con una metodología de corte experimental, incluyendo seguimientos para valorar si los resultados de la intervención perduran a lo largo del tiempo.

\section{Referencias}

Abad-Mas, L., Ruíz-Andrés, R., Moreno-Madrid, F., Sirera-Conca, M. A., Cornesse, M., Delgado-Mejía, D. et al. (2011). Entrenamiento de funciones ejecutivas en el trastorno por déficit de atención/hiperactividad. Revista de Neurología, 52(1), 77-83.

Aguilar, M., Marchena, E., Navarro, J. I., Menacho, I. y Alcalde, C. (2011). Niveles de dificultad de la conciencia fonológica y aprendizaje lector. Revista de logopedia, foniatría y audiología, 31(2), 96-105.

Artiles, C. y Jiménez, J. E. (2006). Programa para la atención educativa al alumnado con TDAH. Canarias: Consejería de Educación, Universidades, Cultura y Deportes del Gobierno de Canarias. Dirección General de Ordenación e Innovación Educativa.

Booth, J. N., Boyle, J. M. E. y Kelly, S. W. (2014). The relationship between inhibition and working memory in predicting children's reading difficulties. Journal of Research in Reading, 37(1), 84-101.

Cuetos, F., Rodríguez, B., Ruano, E., y Arribas, D. (2007). Batería de evaluación de los Procesos Lectores-Revisada Prolec- $R$-. Madrid: TEA Ediciones.

Fernández-Amado, Mª L., Mayor, M. A., Zubiauz, B., Tuñas, A. y Peralbo, M. (2006). Aplicación informática para la evaluación de la conciencia fonológica y competencia lectora. Comunicación presentada en el III Congreso Internacional de lectoescritura. AMEI-WAECE Asociación Mundial de Educadores Infantiles. Morelia - México, 23 25 marzo de 2006.

García, T., González-Castro, P., Rodríguez, C., Cueli, M., Álvarez, D. y Álvarez, L. (2014). Alteraciones del funcionamiento ejecutivo en el trastorno por déficit de atención con hiperactividad y sus subtipos. Revista Psicología Educativa, 20, 23-32.

Greven, C. U., Rijsdijk, F. V., Asherson, P. y Plomin, R. (2012). A longitudinal twin study on the association between ADHD symptoms and reading. Journal of Child Psychology and Psychiatry, 53(3), 234-242.

Jacobson, L. A., Ryan, M., Martin, R. B., Ewen, J., Mostofsky, S. H., Denckla, M. B. et al. (2011).Working memory influences processing speed and reading fluency in ADHD. Child Neuropsychology, 17(3), 209-224.

Katz, L. J., Brown, F. C., Roth, R. M. y Beers, S. R. (2011). Processing speed and working memory performance in those with both ADHD and a reading disorder compared with those with ADHD alone. Archives of Clinical Neuropsychology, 26, 425-433.
Martínez, J. (2014). Relación entre funciones ejecutivas, conciencia fonológica y lectura inicial, en el alumnado del $1^{\circ}$ curso de Educación Primaria. Revista Educación y Futuro Digital, 10, 65-80.

Mayor, M. A., Zubiauz, B., Peralbo, M., Fernández-Amado, Ma. L., Tuñas, A. y Brenlla, J. C. (2011). LOLE: From oral to written language. An instruction program for phonological awareness in kindergarten. Poster presentado en el 12th International Congress for the Study of Child Language. Montreal (Canadá), 19 al 23 de Julio.

Miranda-Casas, A., Fernández, M. I., Robledo, P. y García-Castellar, R. (2010). Comprensión de textos de estudiantes con trastornos por déficit de atención/hiperactividad: ¿qué papel desempeñan las funciones ejecutivas? Revista de Neurología, 50(3), 135-142.

Mueller, S. T. y Piper, B. J. (2014). The Psychology Experiment Building Language (PEBL) and PEBL Test Battery (Version 0.14) [Software]. Avaliable from http://pebl.sourceforge.net

Orjales, I. y Polaino, A. (2001). Programas de intervención cognitivo-conductual para niños con déficit de atención con hiperactividad. Madrid: CEPE.

Thorell, L. B. y Nyberg, L. (2008). The Childhood Executive Functioning Inventory (CHEXI): a new rating instrument for Parents and Teachers. Developmental Neuropsychology, 33(4), 536-552. 